\title{
Electron Mediated Mn-Mn Interaction in Quantum Dots
}

\author{
Z. BAK*
}

Institute of Physics, Jan Długosz University of Częstochowa, al. Armii Krajowej 13/15, 42-200 Częstochowa, Poland

We present a theoretical study of magnetic interaction in quantum dots with magnetic $\mathrm{Mn}^{2+}$ in dot impurities. We show that gate voltage applied to the quantum dot shifts the centers of electron clouds. Exact formulae for the perturbed spin density allow us to derive expression for the change of the strength of the $s p-d$ coupling. Estimations show that $s p-d$ exchange integral is very sensitive to the gate voltage variations. Exact formulae for the change of the effective exchange integrals are derived. As the spin coded qubits are elements of the RAM memory, a part of the energy stored in magnetic coupling will be dissipated when information is written or erased. We estimate this energy and find it sufficiently large to destroy quantum coherence during quantum computing. Finally, we discuss the interdot spin coupling and show the effect of gate voltage operations on the spin intra- and interdot Ruderman-Kittel-Kasuya-Yosida coupling.

PACS: $75.75 .-\mathrm{c}, 75.85 .+\mathrm{t}, 85.75 .-\mathrm{d}$

\section{Introduction}

Studies of the magnetic interactions in systems of reduced geometry are interesting of their own right since a low dimensional system forms a kind of laboratory in which some quantum effects can be tailored by proper choice of boundary conditions. For quantum computing the most promising are the quantum dot (QD) systems as they allow control of a localized single spin [1]. This allows fabrication of spintronic devices in which a qubit represents quantum information by spin orientation of the solid cell. Usually such a cell is composed of two magnetically coupled QDs with spins trapped in them. Tunable spin interaction necessary for logic operations on a qubit opens the prospect of developing spintronic devices which combine information processing and information storage functionalities in one material. Semiconductor-based QDs confine a well-defined number of electrons which determine their total spin. However, one can incorporate a magnetic ion (as the rule this is the Mn ion) into a QD. Usually $\mathrm{Mn}^{2+}$ in its $\left|M^{z}=5 / 2\right\rangle$ spin configuration are used as magnetic impurities. The $s p-d$ coupling induces a variety of physical phenomena like carrier-mediated magnetism, the Zeeman splitting or formation of magnetic polarons and plays the crucial role in the development of spintronic devices based on diluted magnetic semiconductors. As localized ionic spins can be operated by the gate voltage this opens a new possibility for quantum computing. In this case detailed knowledge of magnetic interactions in a controlled electronic environment is essential since $s p-d$ exchange interaction results in a zero field splitting of electric current through the QD.

\section{Electric field vs. the $s p-d$ coupling}

In our contribution we present a study of the influence of gate voltage on the carrier mediated magnetic in-

\footnotetext{
* e-mail: z.bak@ajd.czest.pl
}

teraction among ionic spins placed within a disc-shaped QD. We point out that although there are advanced techniques of positioning of magnetic impurity ions [2], the external, gate electric field always shifts the centers of the QD electron clouds with respect to the magnetic ion positions. This in turn causes a change of the effective exchange integrals between impurity and electron spins. For simplicity we will limit our consideration to disc-like QDs with lateral extension much exceeding its thickness. In this case the electron mobility can be assumed as two-dimensional, while the electron in dot confinement with a good approximation can be modeled by a parabolic potential. With the assumptions above the electron Hamiltonian $H$ can be written in a form which resembles a two-dimensional harmonic oscillator [3]. During quantum logic operations on the qubits there arises contribution from the external electric field. Suppose we apply the electric field $F$ along " $x$ "-axis then we have

$$
H=\left(p_{x}^{2}+p_{y}^{2}\right) /\left(2 m_{\mathrm{e}}\right)+m_{\mathrm{e}} \omega_{0}^{2}\left(x^{2}+y^{2}\right) / 2+e F x,
$$

where $m_{\mathrm{e}}$ is the effective mass of an electron while $\omega_{0}$ is the strength of the parabolic confinement.

The electron eigenstates in our dot are given by the products of the eigenfunctions of the one-dimensional shifted harmonic oscillator [3]:

$$
\begin{aligned}
& \Psi_{n_{x} n_{y}}(x, y)=\Phi_{n_{x}}(x+\beta) \Phi_{n_{y}}(y), \\
& n_{x}, n_{y}=0,1, \ldots
\end{aligned}
$$

where $\Phi_{n_{i}}\left(x_{i}\right)=A_{n} \exp \left(-\alpha^{2} x_{i}^{2}\right) H_{n_{i}}\left(\alpha x_{i}\right), x_{i}=x, y$, with $H_{n_{i}}\left(\alpha x_{i}\right)$ being the Hermite polynomials while $\alpha=\sqrt{m_{\mathrm{e}} \omega_{0} / \hbar}$. The parameter $\beta$ represents the shift of the electron cloud center under action of the external electric field $F$ resulting from the applied gate voltage $V_{\mathrm{g}}$. As $F=V_{\mathrm{g}} / D$, where $D$ is the diameter of the QD the value of each electron cloud shift equals $\beta=e F /\left(m_{\mathrm{e}} \omega^{2}\right)=e V_{\mathrm{g}} /\left(m_{\mathrm{e}} \omega^{2} D\right)$ [4]. The energy spectrum has the well-known form $\epsilon_{n_{x}, n_{y}}=\hbar \omega_{0}\left(1+n_{x}+n_{y}\right)$.

The magnetic moment $M$ of the $\mathrm{Mn}^{2+}$ ion being in the $\left|M^{z}=5 / 2\right\rangle$ state, located at the position $\boldsymbol{R}_{m}=$ $\left.R_{m}^{x}, R_{m}^{y}\right)$ interacts with the free electron spins. The 
$s p-d$ coupling between an electron and $\mathrm{Mn}^{2+}$ spin $S_{\mathrm{M}}$ is given by $H_{\mathrm{el}-\mathrm{Mn}}=-\lambda S_{\mathrm{e}}\left(R_{m}\right) S_{\mathrm{M}} \delta\left(r-R_{\mathrm{M}}\right)$ where $\lambda$ is the $e-\mathrm{Mn}^{2+}$ exchange parameter, while the $S_{\mathrm{e}}$ is the electron spin. The spin density at $R_{\mathrm{M}}$ is given by $s_{\mathrm{e}}=\Sigma_{i \sigma} \Sigma_{j \sigma^{\prime}}\left[\psi_{\mu \sigma}^{*}\left(R_{m}\right) \psi_{\nu \sigma^{\prime}}\left(R_{m}\right)\right]$ (the summation goes over all occupied electron states (2)). Thus, in view of the above the Ruderman-Kittel-Kasuya-Yosida (RKKY) $e-\mathrm{Mn}$ interaction strength is proportional to the spin exchange matrix elements (for details see [5]):

$$
J_{i j}\left(R_{m}\right)=J_{\mathrm{c}}^{2 \mathrm{D}} \psi_{i}^{*}\left(R_{m}\right) \psi_{j}\left(R_{m}\right),
$$

where $J_{\mathrm{c}}^{2 \mathrm{D}}$ is the strength of the in dot $s p-d$ interaction and can be approximated by $J_{\mathrm{c}}^{2 \mathrm{D}}=2 J_{\mathrm{c}} / R_{\mathrm{D}}$, where $J_{\mathrm{c}}$ is the bulk exchange constant and $R_{\mathrm{D}}$ is the QD thickness [5].

In the case of applied electric field this expression changes to

$$
\hat{J}_{i j}^{\mathrm{F}}\left(R_{m}\right)=J_{\mathrm{c}}^{2 \mathrm{D}} \Phi_{i}^{*}\left(R_{m}\right) \Phi_{j}\left(R_{m}\right) .
$$

To estimate the change of the effective exchange interaction that arises from the electron cloud shift let us rewrite the solutions (2) in polar coordinates

$$
\begin{aligned}
& \Psi_{n, m}(r, \theta)=N_{n}^{m} R_{n, m}(r) \mathrm{e}^{-\alpha r^{2}} \mathrm{e}^{\mathrm{i} m \theta}, \\
& n, m=0,1, \ldots
\end{aligned}
$$

where $R_{n, m}(r)$ is a polynomial of order $n$ and $N_{n}^{m}$ is the normalizing factor. Each polynomial $R_{n, m}(r)$ has $n$ zeroes, thus it is reasonable to assume that contributions (see [6]) of different wave functions to the exchange integral - that arise after substitution $r \rightarrow r+\beta$ in their arguments - compensate each other. However, there is the common monotonic component $\exp \left(-2 \alpha^{2} r^{2}\right)$ in each matrix element (4). Under the shift of electron cloud this gives us

$$
J_{i j} \rightarrow J_{i j}^{\prime}=J_{i j} \exp \left(-4 \alpha^{2} R_{m}^{x} \beta-2 \alpha^{2} \beta^{2}\right) .
$$

Since all matrix elements (6) show the same dependence the $s p-d$ exchange integral $J$ inherits the exponential decay and its dependence on electrical field can be written as $J(F)=J_{0} \mathrm{e}^{-\gamma F-\rho F^{2}}$ with $\gamma=4 \alpha^{2} D_{x} /\left(m_{\mathrm{e}} \omega^{2}\right)$ and $\rho=2 \alpha^{2}\left(e /\left(m_{\mathrm{e}} \omega^{2}\right)\right)^{2}$. As we can see, an important effect of the confinement of the magnetic interaction is the change of short range contact interactions. The shrinking of the electron wave functions causes increase of the charge density and this in turn modifies the magnetic interaction. However, the electron density is very sensitive to the external electric field, thus the magnetic interactions become sensitive to applied gate voltage.

Calculation of the magnitude of this shift requires explicit calculations of the parameters $\alpha, \beta, m_{\mathrm{e}} \omega^{2}$. Estimations for the InAs/GaAs(matrix) QD of diameter $D=20 \mathrm{~nm}$ with $V_{\mathrm{g}}=0.5 \mathrm{~V}$ [7] show that the electron cloud shift $\beta$ is of order of a few nanometers [4]. This allows us to estimate fluctuations of the RKKY exchange integrals when external electric field $F=V_{\mathrm{g}} / D$ is varied.

During logic operations on a QD qubit, in each writing/rewriting cycle, the energy of the order of

$$
\epsilon_{\mathrm{F}}=J(F=0)-J(F)=J_{0}-J_{\mathrm{F}}
$$

$$
\propto J_{0}\left[1-\exp \left(-4 \alpha^{2} R_{m}^{x} \beta-2 \alpha^{2} \beta^{2}\right)\right] \approx J_{0} / 2
$$

is dissipated.

Suppose the exchange integral $J_{0}=k T$ is of order of $5 \mathrm{~K}$. If a QD contains, let us say, $10^{4}$ atoms with only one magnetic impurity a single reversal of the ionic spin dissipates energy $\epsilon_{\mathrm{F}} \approx 2 \times 10^{-4} \mathrm{eV}$. The frequency of spin operations in the quantum computers must exceed the frequency limited by the QD spin decoherence time. In the InAs/GaAs(matrix) based QD the spin decoherence time $T_{2}$ equals $2 \mathrm{~ns}$ [8], which means that the spin flipping frequency must be of order of $10^{8} \mathrm{~Hz}$. If we multiply the energy $E$ dissipated in a single flop process $\epsilon_{\mathrm{F}}$ by computing frequency $\omega_{\text {comp }}=10^{8} \mathrm{~Hz}$ we can see that dissipated energy in a second of computing time is $2 \mathrm{eV}$ per atom. This means that it is sufficiently large to destroy quantum coherence of the spintronic cell. It is worth noting that energy dissipation due to the magnetic mechanism is accompanied by dissipation due to parasitic electric dipoles of the QD [4]. Both contributions may lead to the loss of quantum coherency during quantum computing due to the overheating of the QD-qubits. Fortunately, the energy dissipated due to the electric dipoles can be reduced by proper orientation of gate electrodes.

As we know, electric fields applied to the double QD qubit system generate spontaneous electric dipoles that interact with each other. In each writing/erasing cycle the energy

$$
U=\frac{\boldsymbol{p}_{1} \boldsymbol{p}_{2}}{r^{3}}-\frac{\left(3 \boldsymbol{p}_{1} \boldsymbol{r}\right)\left(\boldsymbol{p}_{2} \boldsymbol{r}\right)}{r^{5}}
$$

is dissipated, however, dissipation of energy via this mechanism can be minimized. Indeed, suppose that the gate voltages at both QDs are oriented in such a way that resulting electric fields $\boldsymbol{F}_{1}$ and $\boldsymbol{F}_{2}$ form an angle around $\theta_{0}=\arccos (1 / \sqrt{3})$ with respect to the vector $\boldsymbol{r}$ which joins the centers of adjacent QDs. In this case, in view of Eq. (8), we can draw the conclusion that in such case the dissipation of the energy due to parasitic electric dipoles will be minimized. Contrary to this case orientation at angle the $\theta_{m}=\pi / 2$ (see Fig. 1) increases amount of dissipated energy due to the parasitic dipoles. Unfortunately, there is no idea to minimize dissipation of energy due to magnetic interactions.

\section{Intradot and interdot RKKY interaction}

As it is possible to incorporate a few magnetic ions in a QD, there arises an indirect RKKY coupling between localized spins. The ascertainment is important as the basic difference between RKKY interaction in bulk solids and RKKY interaction in a QD is in the latter being mediated by the confined electrons. As we have shown earlier in confined electron systems the effective density of free electron states $n(\epsilon)$ is often given by a simple power law formula

$$
n(\epsilon) \mathrm{d} \epsilon \approx\left(\epsilon-\epsilon_{0}\right)^{D_{\mathrm{s}} / 2-1} \mathrm{~d} \epsilon .
$$

Parameter $D_{\mathrm{s}}$ represents the effective spectral dimension 


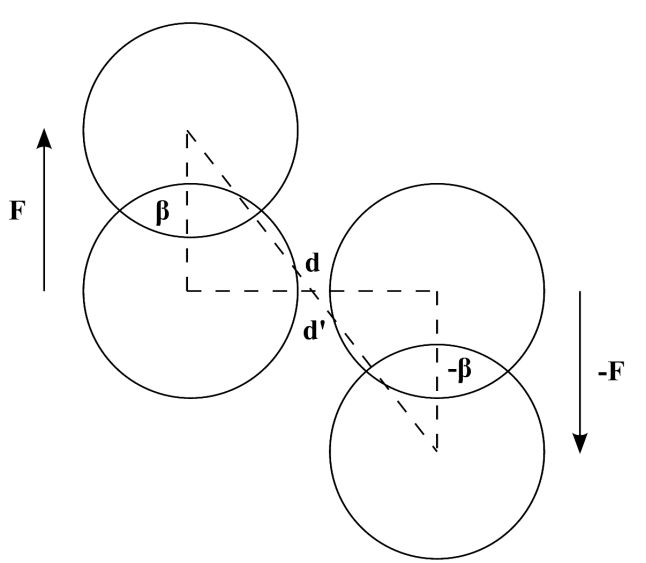

Fig. 1. Shift of the electron cloud in two adjacent QDs in the case of antiparallel electric field.

which in many cases, is a fraction [9]. The RKKY exchange integral in the case is given by [10]

$$
J(r)=J_{0} \xi^{2 \lambda-2}\left[J_{\lambda-1}(\xi) Y_{\lambda-1}(\xi)+J_{\lambda}(\xi) Y_{\lambda}(\xi)\right],
$$

where $J_{\lambda}(\xi)$ and $Y_{\lambda}(\xi)$ are the Bessel and the Neumann functions respectively, while $\xi=\kappa r$ and $\lambda=D_{\mathrm{s}} / 2$. Evidently since the RKKY exchange integral depends on the value of the $s p-d$ exchange integral the intradot exchange integrals also depend on the external electric field via formula (6).

In practical realization the simplest case of the qubit spin coded device contains two magnetically coupled quantum dots with electron spins trapped in them. Logical operations on such a qubit require modulations of the interdot magnetic exchange [1]. That is why we should consider the interdot spin interaction, too. The interdot RKKY interaction is mediated by the free electrons of the lead that joins the adjacent QD of a qubit. As it was shown earlier [10], the electron density is also given by the formula (9). This means that the RKKY interaction is given by the formula (10), however, one should note that due to the different geometry of the lead the parameter $D_{\mathrm{s}}$ in this case takes another value [11]. The most interesting thing is that the interdot RKKY exchange integral also shows dependence on the external electric field. However, the mechanism of this influence is somewhat different [4].

The shift of the electron (spin) clouds is important since RKKY interaction oscillates with the interspin sep- aration favoring either ferro- or antiferromagnetic ordering. With applied external field the distance between QD spins is changed (see Fig. 1). The estimated value of $\beta$ [10] is large enough to switch the RKKY integral from negative to positive (or vice versa). This effect offers the new possibility to control magnetic interactions [10], by changing the distance of QDs spins.

\section{Summary}

We have shown the dependence of the $s d-p$ interaction as well as the $\mathrm{Mn}^{2+}$ RKKY interaction of the gate voltage. We have proved that variations of the effective $s d-p$ as well as RKKY exchange integrals lead to energy dissipation which is sufficiently high to destroy quantum coherence during quantum computing.

As to the practical implications of our study let us note that although the main results were derived for the special system it seems plausible that predicted effects can be realized in any case of Mn doped QD systems. We believe that our study through the derivation of analytical results can be of considerable help for experimentalists when rapid and accurate estimates of magnetic exchange integrals or induced electric dipoles are needed. Finally, we hope that by pointing out unwanted effects our results can be helpful in optimization and tailoring of properties of spintronic devices.

\section{References}

[1] D. Stepanenko, N.E. Bonsteel, D.P. DiVincenzo, G. Burkard, D. Loss, Phys. Rev. B 68, 115306 (2003).

[2] A.M. Yakunin, A.Yu. Silov, P.M. Koenrad, J.H. Wolter, W. Van Roy, J. De Boeck, J.-M. Tang, M.E. Flatte, Phys. Rev. Lett. 92, 216806 (2004).

[3] W. Xie, Q. Xie, Physica E 404, 1625 (2009).

[4] Z. Bak, Acta Phys. Pol. A 118, 957 (2010).

[5] F. Qu, P. Hawrylak, Phys. Rev. Lett. 96, 157201 (2006).

[6] F. Qu, C. de Almeida, P.C. Morais, J. Magn. Magn. Mater. 320, e412 (2008).

[7] E.J. Peter, Phys. Lett. A 355, 59 (2006).

[8] S. Kashiwada, T. Matsuda, K. Yoh, J. Cryst. Growth 301-302, 785 (2007).

[9] Z. Bak, Phys. Rev. B 68, 064511 (2003).

[10] Z. Bak, R. Jaroszewicz, W. Gruhn, Acta Phys. Pol. A 115, 342 (2009).

[11] F. Qu, P. Vasilopoulos, Phys. Rev. B 74, 245306 (2006). 